\title{
Dust and super star clusters in NGC 5253^
}

\author{
L. Vanzi ${ }^{1}$ and M. Sauvage ${ }^{2}$ \\ ${ }^{1}$ ESO - European Southern Observatory Alonso de Cordova, 3107 Santiago, Chile \\ 2 Service d'Astrophysique, CEA/DSM/DAPNIA, Centre d'Études de Saclay, 91191 Gif-sur-Yvette Cedex, France \\ e-mail: msauvage@cea.fr
}

Received 8 July 2003 / Accepted 14 November 2003

\begin{abstract}
We present new observations of the famous starburst galaxy NGC 5253 which owes its celebrity to possibly being the youngest and closest starburst galaxy known. Our observations in the infrared and millimeter contribute to shed light on the properties of this interesting object. We have used our new data along with data from the literature to study the properties of the young stellar clusters present in NGC 5253. We find that the brightest optical clusters are all characterized by a near-infrared excess that is explained by the combined effect of extinction and emission by dust. For the brightest infrared cluster we model the spectral energy distribution from the optical to the radio. We find that this cluster dominates the galaxy emission longward of $3 \mu \mathrm{m}$, that it has a bolometric luminosity of $1.2 \times 10^{9} L_{\odot}$ and a mass of $1.2 \times 10^{6} M_{\odot}$, giving $L / M \approx 10^{3}$. The cluster is obscured by 7 mag of optical extinction produced by about $1.5 \times 10^{5} M_{\odot}$ of dust. The dust properties are peculiar with respect to the dust properties in the solar neighbourhood with a composition characterized by a lack of silicates and a flatter size distribution than the standard one, i.e. a bias toward larger grains. We find that NGC5253 is a striking example of a galaxy where the infrared-submillimeter and ultraviolet-optical emissions originate in totally decoupled regions of vastly different physical sizes.
\end{abstract}

Key words. galaxies: starburst - dust, extinction - infrared - super-star clusters - galaxies: individual: NGC 5253

\section{Introduction}

It has been found that very young clusters can be deeply embedded in dusty cocoons even when hosted in extremely low metallicity environments. The most prominent example of this class of objects is certainly the Super-Star Cluster (SSC) observed in the extremely metal deficient blue compact galaxy SBS 0335-052 (Thuan et al. 1999; Hunt et al. 2001). Modeling the SED of this object indicates that the radiation from a few $\mu \mathrm{m}$ to the far-infrared (FIR) comes from a luminous $\left(>10^{9} L_{\odot}\right)$, massive $\left(>10^{6} M_{\odot}\right)$ star cluster embedded in a dust cocoon where large grains dominate, contrary to interstellar medium dust (Plante \& Sauvage 2002). The amount of dust derived by fitting the SED, about $10^{5} M_{\odot}$, is surprisingly high for a very low metallicity environment and it opens several interesting questions about the star formation history of the galaxy and the processes involved in dust formation. Clusters with similar properties and possibly even more embedded, since they are not detected at $\sim 2 \mu \mathrm{m}$, have been observed in the blue compact galaxy He 2-10 (Vacca et al. 2002). It is found that these clusters have properties very similar to those of galactic

Send offprint requests to: L. Vanzi, e-mail: lvanzi@eso.org

* Based on observations obtained at the ESO telescopes of La Silla and Paranal, program 69.B-0345; and on observations with ISO, an ESA project with instruments funded by ESA Member States (especially the PI countries: France, Germany, The Netherlands and the United Kingdom) and with the participation of ISAS and NASA. ultra dense HII regions and that they are probably the main source of the observed far-infrared flux. In SBS 0335-052, the dust-enshrouded SSC contains about 10 times more O7V stars than its visible counterparts (Plante \& Sauvage 2002). In He 2-10, the embedded sources represent $>1 / 9$ th of the total UV-emitting star content of the galaxy (Kobulnicky \& Johnson 1999; Conti \& Vacca 1994). In these two galaxies, the visible super-star clusters account for almost all of the recent visible star formation activity (Conti \& Vacca 1994; Thuan et al. 1997). Therefore these two examples show that a substantial, if not dominant, fraction of the current star formation can be made invisible in the optical-UV by dust obscuration even in low-metallicity environments. Were this to be a general property, it would obviously have important consequences on the interpretation of the high redshift universe where most star formation should occur in low-metallicity environments.

In this paper, we study the case of NGC 5253, with the purpose of demonstrating that it is also a member of the class of galaxies that host dust-enshrouded SSCs and to strengthen the relevance of the phenomenon in the study of starburst objects.

The starburst galaxy NGC 5253 is a blue dwarf irregular galaxy which has gained increasing interest during the years because of a few important facts. (1) Among the starburst galaxies it is one of the closest examples. Its exact distance is still a matter of debate, with the most recent values being either $3.3 \pm 0.3 \mathrm{Mpc}$ (Gibson et al. 2000) or $4.0 \pm 0.3 \mathrm{Mpc}$ (Thim et al. 2003). Here we arbitrarily choose to use a distance of $3.3 \mathrm{Mpc}$, 
for which $1^{\prime \prime}$ is $16 \mathrm{pc}$. (2) It is most likely the youngest starburst known with a large number of clusters detected whose ages are in the 2-50 Myr range (see e.g. van den Bergh 1980; Gorjian 1996; Calzetti et al. 1997). (3) Although its metal content is not extremely low, as often observed in dwarf galaxies, its metallicity is sub-solar, about $1 / 6 Z_{\odot}$ (Kobulnicky et al. 1999). The starburst nature of this galaxy finds a direct confirmation in the supernovae observed. Two SNe have exploded in less than a century, in 1895 and in 1972, which is a fairly high number given that NGC 5253 is a small galaxy: its estimated mass is $6 \times 10^{9} M_{\odot}$ (Welch 1970 , scaled to the adopted distance).

As NGC 5253 has been observed by many authors over a wide spectral range for many decades, a precise picture of the galaxy can be built today (see e.g. Caldwell \& Phillips 1989; Martin \& Kennicutt 1995; Calzetti et al. 1997, 1999). We will highlight here only those observations that are relevant to our present work.

Rieke \& Low (1972) observed NGC 5253 at 10 and $20 \mu \mathrm{m}$ as part of their seminal work on infrared photometry of galaxies. Glass (1973) and Moorwood \& Glass (1982) also obtained near and mid-infrared observations and attempted to constrain the spectral energy distribution (SED) in the optical and infrared. Roche et al. (1991), based on their mid infrared spectrum which shows strong [SIV] but undetected [NeII], classified NGC 5253, together with II Zw 40, as a high excitation HII galaxy characterized by a young starburst and by the presence of very massive stars. A more complete mid-IR spectrum was published by Crowther et al. (1999) with ISOSWS, and shows that the spectrum is dominated by [SIV], [NeIII] and [SIII], a common feature of high excitation low-metallicity HII regions observed with ISO (e.g. Contursi et al. 2000 in the SMC). Finally, NGC 5253 is detected in all four IRAS bands (Moshir et al. 1990).

A large number of bright clusters was observed by van den Bergh (1980) and interpreted as the remnant of a powerful episode of star formation. More recently high spatial resolution observations have allowed to focus the attention on a few peculiarities. Calzetti et al. (1997, hereafter C97) studied the properties of the brightest optical clusters detected in the HST images. They use broad and narrow band colors and find that all clusters are young with ages from few to a few tens of Myr. The youngest cluster is obscured by about 9 mag in $V$ and in general there is anti-correlation between the presence of dust, that is observed all over the central part of the galaxy, and the age of the clusters, i.e. the younger the clusters, the higher the extinction. The centimetric radio spectrum is entirely thermal (Beck et al. 1996), which is highly unusual for a galaxy, and Turner et al. (2000) detect a very compact radio source, $1-2 \mathrm{pc}$ in size, which is very bright at $1.3-2 \mathrm{~cm}$. They attribute this source to a nebula ionized by about 400007 stars. The ionizing flux derived from the radio is about two orders of magnitude larger than indicated by the $\mathrm{H} \alpha$ emission. Gorjian et al. (2001) report their Keck telescope detection of a bright infrared source at 11.7 and $18.7 \mu \mathrm{m}$, which they identify with the compact radio nebula.

The paper is organized as follows: in Sect. 2 we describe the new observations on which this work is based. In Sect. 3 we discuss the optical colors of the individual bright clusters in the galaxy and exemplify the impact of dust on these colors. In Sect. 4 we build and model the spectral energy distribution of the brightest source in the galaxy and show that it can be represented by a dust-enshrouded SSC model. The conclusions of our work are summarized in Sect. 5.

\section{Observations}

We have observed NGC 5253 with ISAAC at the ESO-VLT in the $K \mathrm{~s}$ and $L^{\prime}$ near-infrared (NIR) broad bands on April 19, 2002. ISAAC is equipped with a Hawaii $1024 \times 1024$ Rockwell detector for the short wavelengths $(1-2.5 \mu \mathrm{m})$ and with an Aladdin SBRC $1024 \times 1024$ detector for the long wavelengths $(2.5-5 \mu \mathrm{m})$, the scale is 0.148 and $0.071^{\prime \prime} /$ pix respectively. Both observations were obtained under seeing better than $0.4^{\prime \prime}$. The total on source integration time was $5 \mathrm{~min}$ in $K \mathrm{~s}$ and about $30 \mathrm{~min}$ in $L^{\prime}$. We have used the usual nodding technique to subtract the background in the $K$ s band and the nod and chop in $L^{\prime}$. The images have been reduced both with the ESO package Eclipse and with IRAF, the two independent techniques gave perfectly consistent results. The photometric calibration relied on the observation of one photometric standard star. Since the Aladdin detector is affected by a significant non linearity, a correction for this effect has been applied to the $L^{\prime}$ band observations. In both filters we have reached a photometric accuracy better than $3 \%$ where most of the error is due to the non-uniformity across the field. We have checked the consistency of our photometry and calibrations using the data available in the literature. Moorwood \& Glass (1982) and Forbes et al. (1992) provide in their work aperture photometry of NGC 5253 on apertures of 3, 6, 9, 12, 36 and 48" diameter in $K$ s and 7.5, 9 and $12^{\prime \prime}$ diameter in $L$. Using the same apertures on our calibrated images, we find values within less than $0.01 \mathrm{mag}$ of Forbes et al. (1992) and $0.02 \mathrm{mag}$ on average of Moorwood \& Glass (1982) in $K$. In $L$, we can only compare with Moorwood \& Glass (1982), the average difference between the two datasets is less than 0.03 mag even though large individual variations are present that can be attributed to centering differences and to the large error bars of the measures of Moorwood \& Glass (1982).

In Fig. 1 we present our $K \mathrm{~s}$ and $L^{\prime}$ band images, the nomenclature for the clusters detected is as in C97. While all the C97 clusters are detected in the $K \mathrm{~s}$ band, only clusters 1,3 and 5 are clearly detected in $L^{\prime}$. Furthermore we find in $L^{\prime}$ a relatively bright source at about 2.4 arcsec NE of cluster 5 which is not among the bright clusters studied in the optical by $\mathrm{C} 97$ and other authors. Looking in the HST images we found a faint optical counterpart for this source. The same source is detected in our Ks image. We will refer this "new" source as 5a. Very faint extended emission, possibly resolved in 2 sources, is observable in $L^{\prime}$ to the SW of cluster 5 in the area of cluster 4 that however cannot be clearly identified. In Table 1 we present the aperture photometry of the clusters studied by $\mathrm{C} 97$. For the ISAAC photometry, we did not apply aperture corrections as with the NIR PSFs, the apertures used include $>99 \%$ of the flux. In addition to those 6 clusters we report the magnitudes of cluster 5a. The photometry for all clusters has been obtained with the same method used by C97 for consistency 


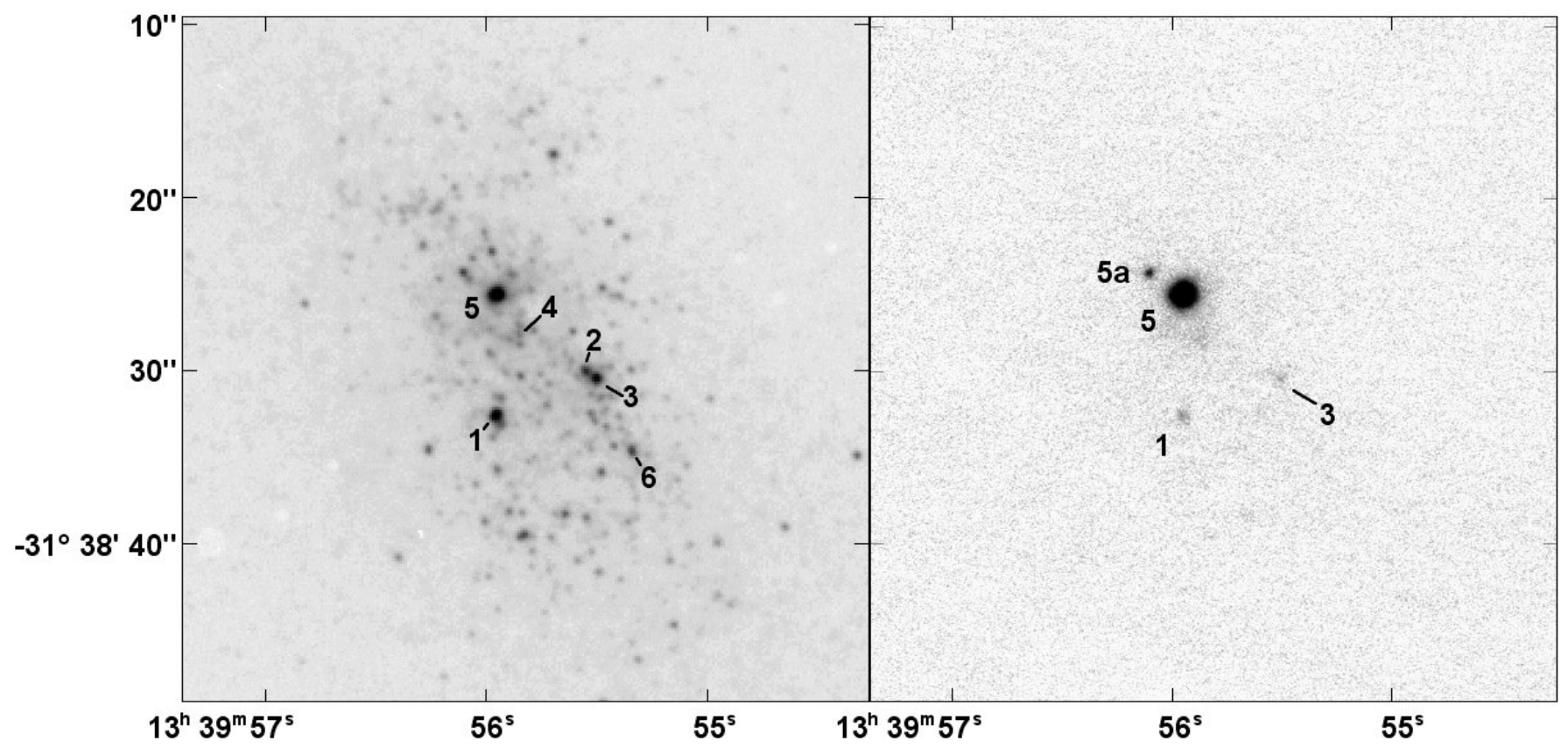

Fig. 1. NGC 5253 in $K$ s (left) and $L^{\prime}$ (right) bands. North is up and East is left. Coordinates refer to the J2000 equinox. The field of view of the image is $40^{\prime \prime}$. The intensity scale on the $K \mathrm{~s}$ band has been chosen to clearly show the brightest clusters. The actual image is much deeper than this display. The clusters are labeled as in C97. As the numbers in C97 correspond to a brightness scale, these images clearly show the dramatic changes occurring in the spectral energy distribution of most clusters in NGC 5253. Cluster 5a is the relatively bright object in the $L^{\prime}$ image North-East of Cluster 5. Note that the intensity levels have been adjusted in the $L^{\prime}$ image to show clusters 1 and 3 , but that cluster 5 is a point source in $L^{\prime}$ as well. Wavelet filtering of the $L$ band image reveals that cluster 4 is responsible for the extension seen SW of cluster 5 , though the statistical significance of this detection is too low to allow a reliable photometric measurement. At an even lower significance level, the same technique indicates the possible detection of a source around $\alpha=13^{\mathrm{h}} 39^{\mathrm{m}} 55.6^{\mathrm{s}}$ and $\delta=-31^{\circ} 38^{\prime} 39^{\prime \prime}$, but at this very low level, this could very well be a false detection.

Table 1. Aperture photometry obtained with a radius of $0.7^{\prime \prime}$ on the main clusters detected in NGC 5253. Typical errors are less than 0.03 mag.

\begin{tabular}{ccc}
\hline \hline & $K_{\mathrm{s}}$ & $L^{\prime}$ \\
\hline 1 & 14.23 & 13.40 \\
2 & 14.60 & - \\
3 & 14.51 & 13.67 \\
4 & 16.10 & - \\
5 & 13.46 & 9.07 \\
$5 \mathrm{a}$ & 15.54 & 12.51 \\
6 & 15.64 & - \\
\hline
\end{tabular}

but with a slightly larger aperture to compensate for the larger Point Spread Function (PSF) of our NIR observations. In the $K$ s band the galaxy background has been measured on an annulus with radius $0.8^{\prime \prime}$ around each source as done by C97 and a width of $0.7^{\prime \prime}$. In $L^{\prime}$ no other source than those listed in Table 1 has been firmly detected, neither is any diffuse emission in the galaxy. Clusters 2 and 3 are separated by less than $1^{\prime \prime}$ so that their $K \mathrm{~s}$ magnitudes are contaminated by each other.

NGC 5253 has been observed at the SEST with SIMBA on the nights of 7 and 8 of June 2002. SIMBA is a 37 channels bolometer array sensitive in the $1.2 \mathrm{~mm}$ band. The beam size at the SEST is about $11^{\prime \prime}$. The background subtraction is achieved by fast scanning of the source through the field of view both in azimuth and altitude. Our maps had a size of $480^{\prime \prime} \times 240^{\prime \prime}$. The atmosphere opacity $\tau$ was very good during all observations with values of about 0.1 . The data have been reduced using the package MOPSI developed by Robert Zylka. As a flux reference we have observed the planet Uranus. The total telescope time on NGC 5253 has been about $3 \mathrm{~h}$ which gave a $1 \sigma$ detection limit of $4 \mathrm{mJy}$. The SIMBA image shows a single unresolved source whose flux is $114 \pm 4 \mathrm{mJy}$.

We complement these observations with the analysis of archival data from the mid-IR camera of the Infrared Space Observatory, ISOCAM ${ }^{1}$. The interest of ISOCAM data is twofold: (1) it has a much higher sensitivity than ground-based instruments operating in the same range, and (2) with a spatial resolution of a few arcseconds, it helps us build a more precise spectral energy distribution for the main cluster of NGC 5253 (see Sect.4). The data analyzed here were part of program LMETCALF.HARO_A and comprise the identification numbers 62500784,62500881 , and 62500980. We analyzed the observations taken with filters LW1-[4.5 $\mu \mathrm{m}]$ to serve as a check on our $L^{\prime}$ observation; LW9-[15 $\left.\mu \mathrm{m}\right]$ to understand the discrepancies found when compiling $20 \mu \mathrm{m}$ photometry (Sect. 4), and LW10-[12 $\mu \mathrm{m}]$ as this filter is equivalent to the IRAS $12 \mu \mathrm{m}$ filter and thus tells us how much of the IRAS flux can be attributed to the central source. All ISOCAM observations were performed with the smallest pixel field-of-view, i.e. 1'.5, in a "beam-switching" mode, i.e. a neighboring empty field is first

\footnotetext{
1 The ISO archive is accessible at
} http: //www. iso.vilspa.esa.es/ 
Table 2. ISOCAM photometry of NGC 5253.

\begin{tabular}{lrrrr}
\hline \hline Filter & $F W H M\left({ }^{\prime \prime}\right)$ & $\lambda_{\text {ref }}(\mu \mathrm{m})$ & $\Delta \lambda(\mu \mathrm{m})$ & Flux density $(\mathrm{mJy})$ \\
\hline LW1 & 2.3 & 4.5 & 1.0 & $125 \pm 30$ \\
LW10 & 3.3 & 12.0 & 7.0 & $1960 \pm 300$ \\
LW9 & 5.1 & 14.9 & 2.0 & $3900 \pm 400$ \\
\hline
\end{tabular}

observed, then the telescope slews to the source. The data were analyzed with CIA V5.0 following the standard procedures. In all 3 bands the source is point-like, which means that the transient-correction scheme used is not adapted (see Coulais \& Abergel 2000 for a discussion). As a result, our fluxes could be lower limits to the actual flux, though at most by $30-50 \%$, given that a very long integration is always performed on the source. The errors quoted in Table 2 are compound errors, i.e. including photometric and transient errors, they are not statistical $1 \sigma$. Fluxes in Table 2 were obtained with aperture photometry correcting for the missed fraction of the PSF, as the ISOCAM PSF has more pronounced low-level wings than the ISAAC one.

\section{Colors of the bright clusters}

We have combined our $K$ s and $L^{\prime}$ observations with those of $\mathrm{C} 97$ to build color-color diagrams of the optically brightest clusters in NGC 5253. For source 5a we measured the optical magnitudes on the HST images using the same technique as in C97. To ensure consistency between our measures we extracted the optical photometry for all clusters from the HST images, the average difference between our values and those of C97 is about 0.02 mag. The F547M-F814W HST color has been converted to $V-I$ using the standard photometric method and a linear fit on the data provided in the HST WFPC2 photometry cookbook ${ }^{2}$. It must be noted that while the F547M magnitudes are quite close to $V$, there is a difference of more than 1 mag. between F814W and $I$ and a large color term. In Fig. 2 we plot the observations (solid circles) in $V-K \mathrm{~s} / V-I$ and $K$ s- $L^{\prime} / V-I$ color-color diagrams. As a reference we also plot the output of Starburst99 (Leitherer et al. 1999) - black solid line - calculated for an instantaneous burst with a metallicity $1 / 5$ solar and standard IMF $\left(2.35,1-100 M_{\odot}\right)$. All observations depart significantly from the model showing a color excess in all bands, the photometric errors on the colors being $0.1 \mathrm{mag}$ at most for the faintest sources. A warning note is due at this point of the discussion. It is well known that sub-solar single stellar population models fail in reproducing the correct blue to red supergiants star ratio (Langer \& Maeder 1995) and that this has important consequences for the predicted colors (Origlia et al. 1999). For this reason, interpretation of Fig. 2 is not free from ambiguities. The problem however only affects the objects in the supergiants phase, cluster 1 and 6 according to the ages derived by $\mathrm{C} 97$. In these cases, the red excess could be artificial and partially or fully due to the lack of red supergiants in the model.

\footnotetext{
${ }^{2}$ http:/www.stsci.edu/instruments/wfpc2/Wfpcc2_phot/ wfpc2_cookbook.html/
}

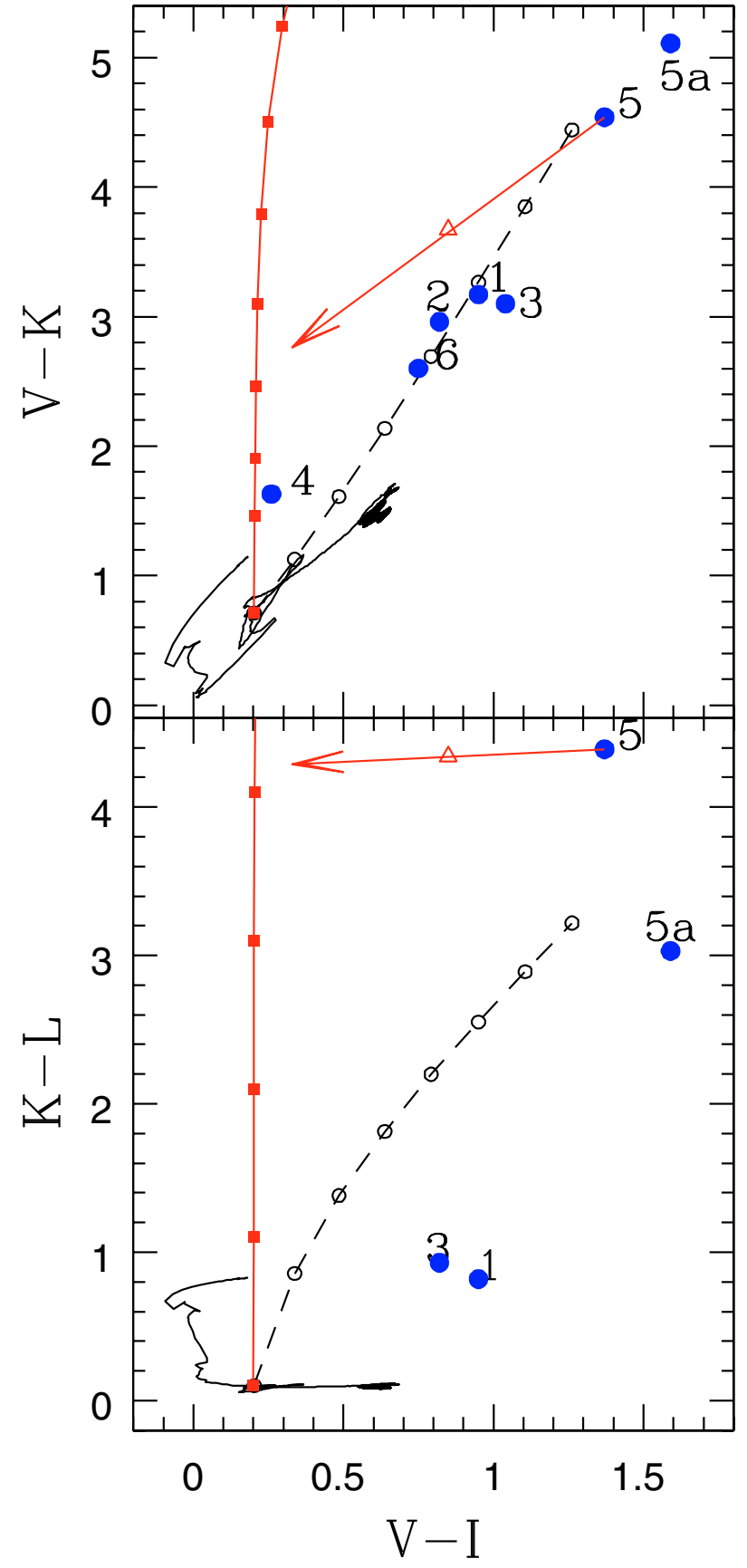

Fig. 2. $V-K / V-I$ and $K-L / V-I$ color-color diagrams of the optically brightest clusters in NGC 5253. The solid black lines represent the output of Starburst99 for a instantaneous burst of $Z=1 / 5 Z_{\odot}$. The solid lines with square points denote the change on the 1 Myr old point introduced by an increasing amount of dust at $1000 \mathrm{~K}$. The arrows indicate the effect of 2 mag of visual extinction on the colors of cluster 5 . The dashed line with open circles shows how a more proper treatment of all dust effects, extinction, scattering and emission, can affect the optical colors. Starting from the $1 \mathrm{Myr}$ dust free Starburst99 model, we compute the colors of the same cluster embedded in a cocoon with the dust properties derived in Sect. 4, with the $V$ optical depth increasing by 1 for each open circle, up to the cluster- 5 best-fit value of 7 . The photometric errors on the data points are of the same size of the symbols or smaller.

To understand the effect that dust can have on the clusters' colors, we examine the color corrections induced by dust 
emission and extinction (a third, more complex effect, i.e. scattering, will be discussed later on). From the colors predicted by the model for an age of $1 \mathrm{Myr}$, the square symbols connected by a continuous line show the effect of adding to the stellar emission an increasing fraction of emission from dust at $1000 \mathrm{~K}$ (treated as a modified blackbody with an emissivity law in $1 / \lambda$ ). The square symbols indicate the fraction of $K \mathrm{~s}$ flux due to dust, with the dust contribution doubling at each step to represent $0,50,67,80,88,94 \%$ etc. of the flux. From cluster 5 we plot a screen extinction vector corresponding to a correction for $A_{V}=2$, an open triagle indicates the value corrected for $A_{V}=1$. We have used high resolution $\left(R=8 \times 10^{4}\right)$ optical spectra obtained with UVES at the VLT and still unpublished, to measure the extinction from the Balmer decrement. Using a screen extinction model, we have obtained $A_{V}=2.65$ and $A_{V}=2.95$ for cluster 1 and 5 respectively. Dust may not present itself only as a screen but could also be mixed with the stars, although this geometry may not be too plausible in the case of clusters. In the mixed case, the attenuation factor is $\left(1-\mathrm{e}^{-\tau_{\lambda}}\right) / \tau_{\lambda}$. The color correction terms that result saturate at high optical depth to values dictated by the extinction curve that are too small to account for the observed clusters' colors. The screen extinction vector thus represents the maximum extinction effect.

Both color-color diagrams give consistent results. The simple examination of Fig. 2 shows that the infrared excess can be explained by the combined effect of extinction and emission by warm dust. There is a degeneracy between age, extinction and dust fraction which prevents from accurately defining the properties of the clusters. There are however a few important facts: (1) an extinction of at least 1-2 mag in $V$ affects all clusters with the only exception of cluster 4 ; (2) a dust contribution in $K$ s above $30 \%$ is required to bring the colors of all clusters close to the model prediction (this number is obtained by "sliding" the cluster points along the extinction vector till they intersect the dust emission line); (3) clusters 5 and 5a display the most extreme color excess: they require more than 2 mag of extinction and a dust contribution in $K$ s above $70 \%$. The properties of cluster 5 will be the main subject of the analysis presented in the following.

It must be noted that the broad band magnitudes and colors of young star forming regions are usually strongly contaminated by the presence of emission lines (the gas continuum emission is taken into account by the Starburst99 model). This effect is more evident in $V$ where the contribution of the emission lines can easily exceed 0.5 mag (Thuan et al. 1997; Vanzi 2003). In our case, however, the HST filters F547M and F814W are free from bright emission lines so that the $V-I$ color derived from them can be considered as representative of the continuum. The only band that can be significantly affected by the presence of emission lines is, in our case, $K$ s so that the $V-K$ s colors must be considered as upper limits. Since the main emission line present in the $K$ s band is $\mathrm{Br} \gamma$, the effect can be quantified using the $\mathrm{H} \alpha$ fluxes measured by $\mathrm{C} 97$ and a standard value for the $\mathrm{H} \alpha / \mathrm{Br} \gamma$ ratio. For cluster 5, even assuming an extinction $A_{V}=9$ as derived by C97, we obtain a correction of only $0.08 \mathrm{mag}$. The other clusters have equivalent widths of $\mathrm{H} \alpha$ that are considerably smaller so that the correction for those cases can be considered negligible. Another effect that could potentially affect the $V-K$ s color is the different angular resolution of the HST and VLT images which could be only partially compensated by the different apertures used to extract the photometry.

Anticipating the results presented in Sect. 4, we draw the reader's attention on the apparent disagreement between the rather small visual extinction that can be derived from Fig. 2 and that derived by $\mathrm{C} 97$. The reason is that the extinction vector drawn in Fig. 2, as is generally the case in such graphs, only takes into account the extinction effect, and not the scattering by dust grains. It has long been known (e.g. Witt et al. 1992) that while extinction reddens the intrinsic spectrum, scattering makes it bluer. As a net result, when scattering is taken into account, the magnitude of the extinction vector decreases for the same $A_{V}$. As $C 97$ took scattering into account in their estimation of extinction, they obtain a larger value than in the simple extinction-only case plotted in Fig. 2. On the same figure, we show the color evolution of the model derived in Sect. 4, with an increasing $\tau_{V}$ (open circles, each circle marks an increase by 1 in the $V$ optical depth, up to $\tau_{V}=7$ ). This model completely incorporates the extinction, scattering and emission effects of dust. Because it incorporates both extinction and emission from dust, the orientation of the color correction vector is a combination of those of the emission vector (squares) and screen extinction (triangle) vector. But since it also accounts for scattering, the amplitude of the color correction for one mag. of optical depth is smaller than in the pure screen extinction case. As will be seen in Sect. 4, our model only partially reproduces the $L^{\prime}$ band flux, hence the disagreement in the $K \mathrm{~s}-L^{\prime}$ color.

All clusters are barely resolved in our images. In particular in $K \mathrm{~s}$ where all field stars display a uniform PSF with $F W H M$ of 0.40 , clusters 1 and 6 have a $F W H M$ of 0.50 , cluster 5 of 0.46 , cluster 3 of 0.52 , and cluster 2 of $0 .{ }^{\prime} 60$, though this last value is made uncertain by the proximity of cluster 3. Cluster 4 is too faint to measure a meaningfull $F W H M$. Deconvolving with the stellar PSF we obtain sizes in the range $0.22-0.45(3.5-7.2 \mathrm{pc})$ that are fully consistent with those measured by $C 97$. In the $L^{\prime}$ image there are no stars visible so that we have to rely on the standard stars which have a FWHM

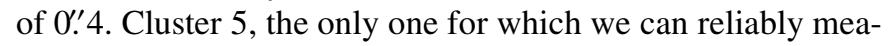
sure the $F W H M$ in $L^{\prime}$, has a FWHM of 0.47 consistent with the value measured in $K \mathrm{~s}$. This means that the dust emission is very compact in $K \mathrm{~s}$ and $L^{\prime}$ and that it is confined to the optically emitting region.

\section{Modeling the SED}

As it is evident from Fig. 2, cluster 5 stands out among the population of bright stellar clusters in NGC 5253. Since cluster 5 sits at the position of the thermal radio nebula of Turner et al. (2000), we can assume that it is indeed the optical counterpart of the radio nebula. The fact that optical studies show it to suffer from strong extinction strengthens this hypothesis. Thus in this section we first compile observations to build the full spectral energy distribution of cluster 5 , from the optical to the radio, and then we attempt to model this SED using the same approach as in SBS 0335-052 (Plante \& Sauvage 2002), i.e. 
modeling the stellar light transfer in the dust envelope with DUSTY (Ivezić \& Elitzur 1997).

\subsection{The SED of cluster 5}

From the optical to the $L^{\prime}$ band our task is simple since the spatial resolution of HST (C97) or VLT (this work) allows to separate the cluster from its neighbors and to measure accurately its fluxes. Our optical photometry of cluster 5 agrees within 0.06 mag with C97, the difference is mostly due to the background subtraction.

In the mid-IR range, fluxes come from a variety of works, from the early ones with photometers of Rieke \& Low (1972), Moorwood \& Glass (1982), and Frogel et al. (1982), to stateof-the-art cameras on large ground-based telescopes (Gorjian et al. 2001) and space observatories, namely IRAS and ISO. At the shortest infrared wavelengths, the ISOCAM $4.5 \mu \mathrm{m}$ flux of $125 \pm 30 \mathrm{mJy}$ compares well with the $77 \mathrm{mJy}$ measured in the $L^{\prime}(3.78 \mu \mathrm{m})$ image, once taken into account the strong rise from $K \mathrm{~s}$ to $L^{\prime}$. There is thus no reason to doubt that all of the ISOCAM $4.5 \mu \mathrm{m}$ flux comes from cluster 5 . Around $10 \mu \mathrm{m}$ the measurement from Rieke \& Low (1972) appears rather high compared with the more recent determinations of both Frogel et al. (1982) and Moorwood \& Glass (1982) and we thus choose to exclude it from the SED. As the SED is seen to increase as the wavelength increases, the 2.2 Jy determination at $11.7 \mu \mathrm{m}$ of Gorjian et al. (2001) can be considered compatible with the rest of the photometry, although on the high side. The comparison of our LW10 and the IRAS $12 \mu$ m flux shows that the ISO flux represents only $\sim 75 \%$ of the IRAS flux. Given that dust is clearly seen to be widespread in the galaxy, we attribute this discrepancy to diffuse dust, to which ISO and ground based telescopes are much less sensitive than IRAS. Diffuse dust, also called cirrus dust in the Galactic context, has its emission peak around $100 \mu \mathrm{m}$. Therefore it must contribute emission to all four IRAS bands, meaning that we cannot attribute $100 \%$ of the IRAS fluxes at 25, 60 and $100 \mu \mathrm{m}$ to cluster 5. As we do not have high spatial resolution measurements at these wavelengths, we assume that the same correction factor applies to them and consider that only $75 \%$ of the IRAS flux at 25,60 and $100 \mu \mathrm{m}$ originates from cluster 5 .

Moving to the range $15-25 \mu \mathrm{m}$, the situation becomes slightly confused. As the $18 \mu \mathrm{m}$ range is visible from the ground, a number of measurements exist, but they show a rather large scatter, from the small value of $2.9 \mathrm{Jy}$ obtained at $18.7 \mu \mathrm{m}$ by Gorjian et al. (2001) to the large IRAS $25 \mu \mathrm{m}$ flux of $9 \mathrm{Jy}$ ( $75 \%$ of $12 \mathrm{Jy}$ ). Even though the wavelengths are not the same, an increase by more than a factor of 4 is quite surprising. In fact we suspect that, although it is backed by some earlier measurements, the Gorjian et al. flux underestimates the actual flux in this range. For instance, the point source detected with ISOCAM at $14.9 \mu \mathrm{m}$ has a flux of $3.9 \pm 0.4 \mathrm{Jy}$, already higher than the $18.7 \mu \mathrm{m}$ flux, and very few sources show such a decreasing spectrum from 14.9 to $18.7 \mu \mathrm{m}$, even when taking into account the second silicate absorption band at $18 \mu \mathrm{m}$. Furthermore, and although the photometric accuracy on the continuum is probably not very good, the spectrum of Crowther et al. (1999) shows that the continuum increases slightly from 15 to $19 \mu \mathrm{m}$, with the level at $25 \mu \mathrm{m}$ being a good factor of 2 higher than at $18 \mu \mathrm{m}$. The low $Q$ band flux of Rieke \& Low (1972) at 3.7 Jy could lend some support to the Gorjian et al. (2001) measurement however Rieke \& Low (1972) give no detail on the band used for this measurement and thus we cannot use it to constrain the SED. According to Gorjian (private communication) the low $18.7 \mu \mathrm{m}$ flux could be due to too small a chopping throw, resulting in an incorrect zero-level, or a complex airmass correction. We note that a similar measurement in the $18.7 \mu \mathrm{m}$ filter was obtained by Martin-Hernandez et al. (2004) giving a higher flux of 5.7 Jy. We will thus discard the $18.7 \mu \mathrm{m}$ measurement of Gorjian et al. (2001) from the SED fit.

Further on in the infrared, we only have IRAS measurements, for which the PSF is almost as large as the galaxy. Again we will attribute only $75 \%$ of the IRAS flux at 60 and $100 \mu \mathrm{m}$ to cluster 5 .

Coming to the submillimeter and millimeter range, we enter a realm where dust continuum emission is not necessarily the dominant emission process. Indeed synchrotron or free-free emission usually dominate longward of a few $\mathrm{cm}$. Here we will follow the computations of Turner et al. (1997). These authors show that longward of $2 \mathrm{~cm}$, and for the compact radio source, the flux can reasonably be considered as pure free-free emission. This process has a spectral index of $\alpha=-0.1$ (in $f_{v} \propto v^{\alpha}$ ). From the $2 \mathrm{~cm}$ flux they compute the remaining dust continuum emission at $2.6 \mathrm{~mm}$, an upper limit of $26 \pm 10 \mathrm{mJy}$. This will be the longest wavelength included in the SED, and given that it is based on interferometric data we attribute all this flux to cluster 5. Using the same method we estimate the free-free contribution in our $1.2 \mathrm{~mm}$ observation and in the James et al. (2002) $850 \mu \mathrm{m}$ SCUBA observation at respectively 43 and $41 \mathrm{mJy}$. For those last two measurements however we are left in a situation intermediate between the optical and the IRAS ones: at $\sim 10-20^{\prime \prime}$, the PSF could be rather large compared to the object. Judging from the James et al. (2002) map, the SCUBA emission comes from the central region of the galaxy, where cluster 5 is located, but also where most of the star formation activity, and ionized gas are concentrated (as revealed e.g. by the radio and $\mathrm{H} \alpha$ images of Turner et al. 1998). This is also the case in our $1.2 \mathrm{~mm}$ observations. The association with the ionized gas could simply reflect the free-free contribution ( $\sim 20 \%$ ) but we have no means of ascertaining that. Therefore we will attribute to cluster 5 the full free-free-corrected fluxes at $1.2 \mathrm{~mm}$ and $850 \mu \mathrm{m}$, i.e. respectively $71 \pm 4$ and $151 \pm 23 \mathrm{mJy}$. The errors quoted here are the formal errors derived from ours or James et al. (2002) photometry. A further source of error comes from the free-free correction, however Turner et al. (1997) do not provide enough details for us to estimate the associated error.

Table 3 and Fig. 3 summarize all the measurements included in the spectral energy distribution of cluster 5 .

Judging from Fig. 1 one could think that the existence of source 5a brings a further uncertainty to the SED we have just built. We do not think that this is the case since already in $L^{\prime}$ the source is $3.5 \mathrm{mag}$ fainter, and is less red than cluster 5 . 


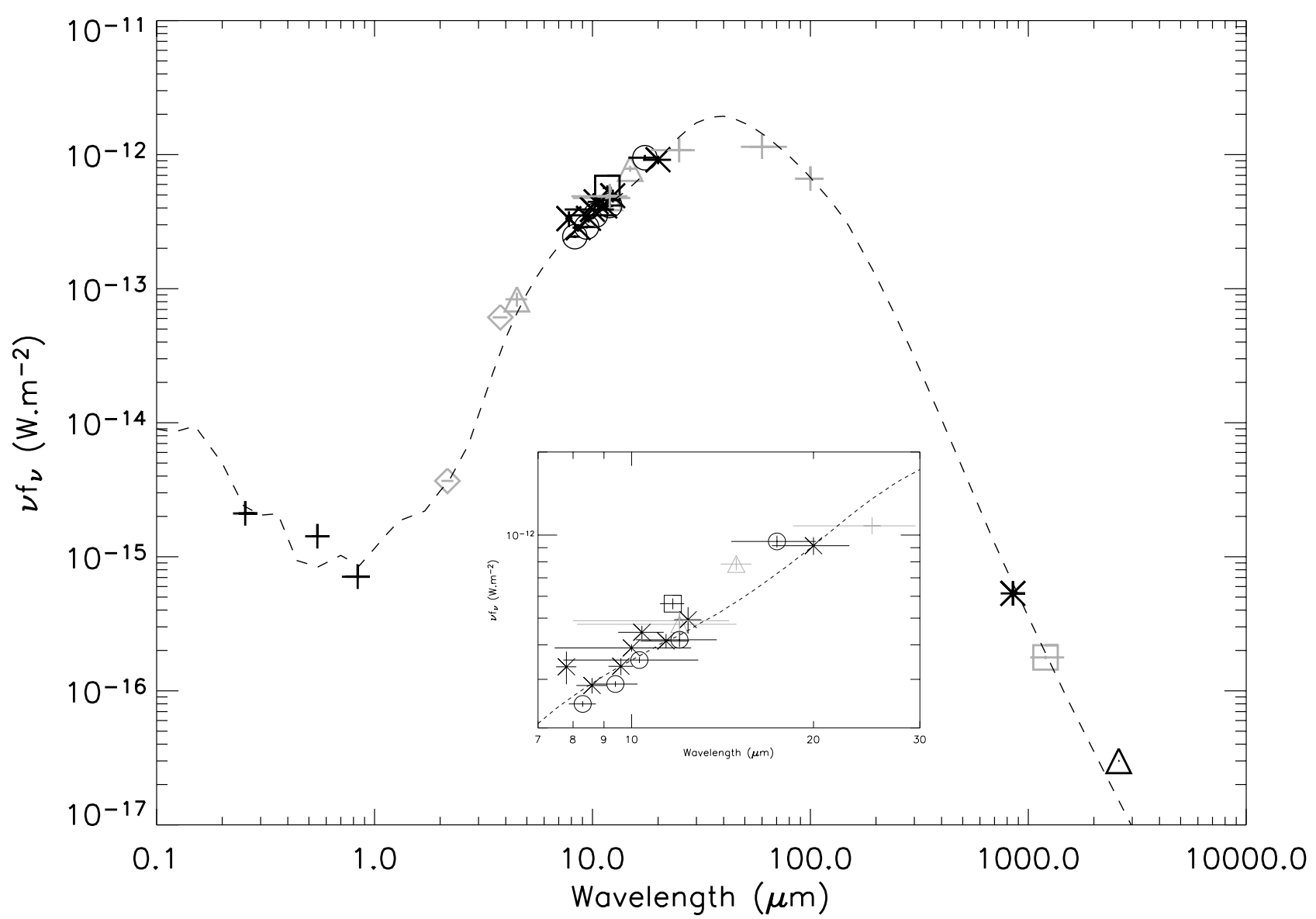

Fig. 3. The spectral energy distribution of cluster 5 in NGC 5253. The 7-30 $\mu \mathrm{m}$ range has been blown up for clarity in the inset. Symbols refer to the photometric source, from left to right: black plus signs correspond to the HST photometry, grey diamonds to the VLT/ISAAC data, grey triangles to the ISO data, black crosses in the $10 \mu \mathrm{m}$ range to the Frogel et al. (1982) data, black circles to the Moorwood \& Glass (1982) data, the black square to the Gorjian et al. (2001) $11.7^{\prime} \mu \mathrm{m}$ point, grey plus signs to IRAS data, the black cross in the submillimeter range to SCUBA data, the grey square to our SEST/SIMBA observation, and the black triangle is the OVRO upper limit. Horizontal error bars describe the filter bandwidths, while vertical error bars show the photometric errors, as quoted by the respective authors (see Table 3). In most cases, these bars are shorter than the symbol size. The dashed line is the best fitting DUSTY model.

Furthermore, it does not appear to be detected with groundbased telescopes at $10 \mu \mathrm{m}$.

\subsection{Fitting the SED}

Now that the spectral energy distribution has been built, we turn to DUSTY (Ivezić \& Elitzur 1997) to model it. The main feature of DUSTY is that it models the radiative transfer of light emitted from a point source inside a spherically symmetric shell of dust. Though straightforward to describe, this problem actually involves a relatively large number of parameters. The first one is obviously the light source. Here, following Sect. 3, we will use Starburst99 (Leitherer et al. 1999) to generate instantaneous burst models with ages from 1 to $9 \mathrm{Myr}$ at a metallicity of $1 / 5 Z_{\odot}$, close to the one of NGC 5253 . Describing the dust shell requires many more parameters. The first of these is the dust equilibrium temperature at the inner side of the shell, $T_{\text {in }}$. Due to the strategy developed by DUSTY to solve the transfer problem, this temperature sets the physical scale of the model. The radial profile of the dust density has to be described. We take it as a broken power law. For $N_{\text {rad }}$ radial zones, this generates $\left(N_{\text {rad }}-1\right)$ radius values for the zone limits as the outer radius is fixed in terms of the inner radius, and $N_{\text {rad }}$ exponents. To introduce some flexibility in the density profile with a limited number of parameters, we chose to run models with two radial zones. In principle, DUSTY can include six different dust grains species. However these are mainly variations around three principal types: the classical astronomical silicates and graphite (Draine \& Lee 1984), and amorphous carbon grains, a possibly more realistic representation of interstellar carbon-based dust. With three grains species, the dust composition introduces 2 parameters. Dust is also characterized by its size distribution, taken as a power-law, and thus described with three parameters, the minimum and maximum grain size, and the exponent of the power law. The standard size distribution, the so-called MRN distribution has $a_{\min }=0.005 \mu \mathrm{m}$, $a_{\max }=0.25 \mu \mathrm{m}(1 \mu \mathrm{m}$ for graphite $)$ and an exponent $q=3.5$ (in the sense $n(a) \propto a^{-q}$, Mathis et al. 1977). This size distribution is such that, for a 50-50 mix of astronomical silicate and graphite, it reproduces the Galactic interstellar extinction curve. Finally, the last parameter describing the shell is its thickness, characterized in DUSTY by the visible optical depth $\tau_{V}$. 
Table 3. The compiled spectral energy distribution of Cluster 5. Aperture size are listed when appropriate (i.e. mostly for single-beam measurements). A few telescopes and filter bandwidths are missing because we were unable to find the information in the original papers.

\begin{tabular}{|c|c|c|c|c|c|c|}
\hline Telescope/instrument & Filter name & $\begin{array}{r}\lambda \\
\mu \mathrm{m}\end{array}$ & $\begin{array}{l}\Delta \lambda \\
\mu \mathrm{m}\end{array}$ & $\begin{array}{r}\text { Aperture } \\
\prime \prime\end{array}$ & $\begin{array}{r}\text { Flux } \\
\text { mJy }\end{array}$ & Source \\
\hline HST/WFPC2 & $F 255 W$ & 0.2574 & 0.041 & & 0.179 & C97 \\
\hline HST/WFPC2 & $F 547 M$ & 0.5465 & 0.049 & & 0.259 & C97 \\
\hline HST/WFPC2 & $F 814 W$ & 0.8370 & 0.176 & & 0.198 & C97 \\
\hline VLT/ISAAC & $K \mathrm{~s}$ & 2.16 & 0.27 & & 2.65 & this work \\
\hline VLT/ISAAC & $L^{\prime}$ & 3.78 & 0.58 & & 77.2 & this work \\
\hline ISO/ISOCAM & $L W 1$ & 4.5 & 1.0 & & $125 \pm 30$ & this work \\
\hline CTIO $1.54 \mathrm{~m}$ & & 7.8 & 0.6 & 8.2 & $866 \pm 234$ & Frogel et al. (1982) \\
\hline ESO $3.6 \mathrm{~m}$ & $N 1$ & 8.3 & 0.85 & 7.5 & $676 \pm 32$ & Moorwood \& Glass (1982) \\
\hline CTIO $1.54 \mathrm{~m}$ & & 8.6 & 1.0 & 8.2 & $817 \pm 106$ & Frogel et al. (1982) \\
\hline ESO $3.6 \mathrm{~m}$ & $N 2$ & 9.4 & 1.65 & 7.5 & $904 \pm 43$ & Moorwood \& Glass (1982) \\
\hline CTIO $1.54 \mathrm{~m}$ & & 9.6 & 0.9 & 8.2 & $1070 \pm 150$ & Frogel et al. (1982) \\
\hline \multirow[t]{2}{*}{ CTIO $1.54 \mathrm{~m}$} & & 10.0 & 5.1 & 8.2 & $1300 \pm 80$ & Frogel et al. (1982) \\
\hline & & 10.0 & 5.4 & 6.0 & $2130 \pm 150^{a}$ & Rieke \& Low (1972) \\
\hline ESO $3.6 \mathrm{~m}$ & $N$ & 10.3 & 5.2 & 7.5 & $1210 \pm 60$ & Moorwood \& Glass (1982) \\
\hline \multirow[t]{3}{*}{ CTIO $1.54 \mathrm{~m}$} & & 10.4 & 1.8 & 8.2 & $1540 \pm 170$ & Frogel et al. (1982) \\
\hline & & 10.4 & 1.3 & 8.5 & $1365 \pm 90$ & Lebofsky \& Rieke (1979) \\
\hline & & 10.6 & 5 & 8.5 & 1500 & Lebofsky \& Rieke (1979) \\
\hline CTIO $1.54 \mathrm{~m}$ & & 11.4 & 2.1 & 8.2 & $1570 \pm 190$ & Frogel et al. (1982) \\
\hline Keck/LWS & & 11.7 & 1.0 & & $2200 \pm 200$ & Gorjian et al. (2001) \\
\hline ESO $3.6 \mathrm{~m}$ & $N 3$ & 12.0 & 3.7 & 7.5 & $1670 \pm 200$ & Moorwood \& Glass (1982) \\
\hline ISO/ISOCAM & $L W 10$ & 12.0 & 7.0 & & $1900 \pm 300$ & this work \\
\hline IRAS & 12 & 12.0 & 7.0 & & $2610 \pm 131^{b}$ & Moshir et al. (1990) \\
\hline CTIO $1.54 \mathrm{~m}$ & & 12.4 & 1.3 & 8.2 & $2040 \pm 450$ & Frogel et al. (1982) \\
\hline ISO/ISOCAM & $L W 9$ & 14.9 & 2.0 & & $3900 \pm 400$ & this work \\
\hline ESO $3.6 \mathrm{~m}$ & $Q$ & 17.4 & 5.6 & 7.5 & $5500 \pm 539$ & Moorwood \& Glass (1982) \\
\hline Keck/LWS & & 18.7 & 0.5 & & $2900 \pm 300^{a}$ & Gorjian et al. (2001) \\
\hline \multirow[t]{2}{*}{ CTIO $1.54 \mathrm{~m}$} & & 20.0 & 5.9 & 8.2 & $6100 \pm 900$ & Frogel et al. (1982) \\
\hline & $Q$ & 21.0 & & 6.0 & $3700 \pm 400^{a}$ & Rieke \& Low (1972) \\
\hline IRAS & 25 & 25.0 & 11.15 & & $12000 \pm 600^{b}$ & Moshir et al. (1990) \\
\hline IRAS & 60 & 60.0 & 32.5 & & $30500 \pm 1220^{b}$ & Moshir et al. (1990) \\
\hline IRAS & 100 & 100.0 & 31.5 & & $29400 \pm 1760^{b}$ & Moshir et al. (1990) \\
\hline JCMT/SCUBA & & 850.0 & 62 & 41 & $151 \pm 23^{c}$ & James et al. (2002) \\
\hline SEST/SIMBA & & 1200.0 & 465 & & $71 \pm 4^{c}$ & this work \\
\hline OVRO & & 2600.0 & 22.6 & & $<26^{c}$ & Turner et al. (1997) \\
\hline
\end{tabular}

${ }^{a}$ This measurement is not used as a constraint for the SED fit, see text for details.

${ }^{b}$ Only $75 \%$ of this flux is used as a constraint for the SED fit, see text for details.

${ }^{c}$ Corresponds to the dust-only flux, see text for details.

Therefore to summarize the parameters that describe a DUSTY model in our case are: $t_{\mathrm{sb}}$ the age of the burst, $T_{\text {in }}$ the temperature on the inner side of the shell, $X_{\mathrm{Si}}$ and $X_{\mathrm{gr}}$ the abundances of silicates and graphite (the abundance of amorphous carbon is $\left.X_{\mathrm{am}}=1-\left[X_{\mathrm{Si}}+X_{\mathrm{gr}}\right]\right), a_{\min }, a_{\mathrm{max}}$, and $q$ the parameters of the size distribution, $r_{\lim }$ the transition radius between the two radial zones, $\beta_{1}$ and $\beta_{2}$ the exponents of the density powerlaw (in the sense $\rho \propto r^{-\beta}$ ), and finally $\tau_{V}$ the optical depth. This is a total of 11 parameters. As can be seen in Table 3 we have almost 3 times as many photometric data points so the problem is clearly over-constrained. However an 11-dimension parameter space is not one that is easy to explore systematically. To identify the best-fit model, we choose a strategy where we start from a standard dust shell model and have a restricted set of parameters explore their full space. The best fitting model among this set is then used as a "seed" and a new set of parameters is allowed to explore a large part of their respective space. In general, when we start with a new seed model, parameters of the previous exploration are still allowed some margin. This is especially true of $\tau_{v}$ for purely technical reasons. This is 
Table 4. Parameters of the best-fitting dusty model. In the acceptable range, we give an indication of the range of values that, while producing a worse fit, still correspond to a model reproducing the data within the photometric uncertainties.

\begin{tabular}{lll}
\hline \hline Parameter & Value & Acceptable range \\
\hline$t_{\mathrm{sb}}$ & $1 \mathrm{Myr}$ & $1-2 \mathrm{Myr}$ \\
$T_{\mathrm{in}}$ & $570 \mathrm{~K}$ & $550-650 \mathrm{~K}$ \\
$X_{\mathrm{Si}}$ & 0 & $<10$ \\
$X_{\mathrm{Gr}}$ & 0.84 & $0.8-0.9$ \\
$a_{\min }$ & $0.005 \mu \mathrm{m}$ & $<0.01 \mu \mathrm{m}$ \\
$a_{\max }$ & $0.5 \mu \mathrm{m}$ & $0.4-0.6 \mu \mathrm{m}$ \\
$q$ & 2.5 & $2.5-3.0$ \\
$r_{\mathrm{lim}}$ & $2.8 \mathrm{pc}$ & $2.8-7 \mathrm{pc}$ \\
$\beta_{1}$ & 0 & $0-0.5$ \\
$\beta_{2}$ & 0.5 & $0-0.5$ \\
$\tau_{\mathrm{V}}$ & 7 & $7-8$ \\
\hline
\end{tabular}

repeated a number of times so that all the 11 dimensions of the parameter space are explored more than once.

To compare a model to the observations, the model SED is integrated using the filters' respective transmission curves and photometric conventions to produce model fluxes. For the photometric data of Moorwood \& Glass (1982) and Frogel et al. (1982), we could not find the actual filter transmissions and thus a square bandpass was used. The best fitting model is the one that minimizes the quantity:

$\Delta=\sum_{i=1}^{N_{\text {filter }}}\left[\frac{f_{i}^{\text {obs }}-f_{i}^{\text {mod }}}{f_{i}^{\text {obs }}}\right]^{2}$.

The advantage of Eq. (1) is that it gives an equal weight to the small and large flux values, an important fact given the very large dynamic range of cluster 5's SED (about 6 orders of magnitude), and that it is symmetric, i.e. models that over- or underpredict the SED by the same amount have equal weight.

The best-fitting model is displayed as a dashed line in Fig. 3. The search for a better fit in an 11-dimension parameter space can be a never-ending one. This model is the bestfitting in the sense that it reproduces the data within the average photometric error, with the exception of the F547M filter. In Table 4 we have indicated the range in which individual parameters can vary without significantly degrading the fit. Before extracting physical information from the fit, it is worth commenting on some of the features discovered as we searched for the best fitting model.

First, while we have allowed the age to vary between 1 and $9 \mathrm{Myr}$ for a substantial part of the parameter space exploration, we have found no intermediate best-fitting model (or "seed" model to use the terminology above) for ages different than 1 or $2 \mathrm{Myr}$. This age is younger than that derived from the optical colors alone, although not by a large amount. It is also smaller than the age of the Antennae dust-enshrouded SSC (Gilbert et al. 2000), making it one of the youngest such source.

Second, the optical depth converges invariably toward a value of 7-8. Interestingly, this is very close to the value measured by $\mathrm{C} 97,9 \mathrm{Vmag}$. It is also in the same range as that measured by Gilbert et al. (2000) for the Antennae SSC. However it does not agree with what can be deduced from the colorcolor diagram presented in Fig. 2: the red arrow extending from cluster 5 seems to imply only $2 \mathrm{Vmag}$ of extinction. The reason is simple: the effect of dust on the flux in the $V, I, K \mathrm{~s}$ and $L^{\prime}$ bands is much more complex than a simple screen extinction on the stellar flux combined with thermal emission in the infrared bands. Scattering also has to be taken into account and, as it is amply demonstrated in Witt et al. (1992), it makes the intrinsic spectrum bluer, and thus works in the opposite direction of extinction. This very complex effect of dust is exemplified in Fig. 2 with the dashed line: we have taken the best fitting model and decreased its visual optical depth $\left(\tau_{V}\right)$ from 7 to almost 0 (i.e. no dust at all). As can be seen, the effect on the colors combines that of the simple screen extinction and hot dust contamination, but the color change per unit of optical depth is much less than that implied by screen extinction, mostly because of scattering.

Finally the temperature on the inner side of the shell is also rather stable in all seed models at around 500-600 K, with the best-fit value at $570 \mathrm{~K}$. When compared with the results obtained for SBS 0335-052 (Plante \& Sauvage 2002), i.e. an optical depth of 30 and an internal temperature of $700 \mathrm{~K}$, this shows that the cluster in NGC 5253 is a less extreme case than that of SBS 0335-052, though not strikingly different.

From the actual parameters of the best-fitting model (see Table 4), we can extract physical parameters of the embedded source and its surrounding cocoon (the equations to perform this can be found in Plante \& Sauvage 2002). The bestconstrained parameter is the luminosity since this is simply the integral of the SED, which is already well described by the observations. The luminosity we obtain is $1.2 \times 10^{9} \mathrm{~L}_{\odot}$. The uncertainty on this value is $\sim 20 \%$. The bolometric luminosity of a nearby galaxy is a very difficult quantity to estimate, however one can get a good insight of the importance of cluster 5 by comparing its SED, i.e. Fig. 3, to the complete SED of the galaxy, readily available on the NASA Extragalactic Database ${ }^{3}$. This shows, quite expectedly, that cluster 5 produces nearly all of the IR-Submm luminosity of NGC 5253, but more important, it produces a luminosity equivalent to that emerging in the UV-visible region. Therefore this shows that a single source, of very modest physical size, can generate as much energy as the complete galaxy. In other terms, it means that even when we consider the global SED of the galaxy, the origin of the energy can be a very strong function of the wavelength, i.e. from the widespread distribution of stars over the whole galaxy body in the UV-visible, to a single compact source in the IR-Submm. Depending on the representativity of NGC 5253 with respect to other galaxies, this fact could have a significant impact on all attempts to model the evolution of global SED (e.g. Chary \& Elbaz 2001; Dale \& Helou 2002; Chapman et al. 2003). Finally, to compare clusters to one another, it is often convenient to express the luminosity as a number of equivalent O7V stars (see the properties of this "standard" in Vacca et al. 1996). We find that the bolometric luminosity of cluster 5 requires 4700 of these stars, well in the range of SSC's stellar content and quite close to the radio estimate of 4000 (Turner et al. 2000).

\footnotetext{
${ }^{3}$ see http://nedwww.ipac.caltech.edu/
} 
Using this luminosity and Starburst99 we can obtain the mass of stars present in the cluster. This is $8.2 \times 10^{5} M_{\odot}$, very close to the estimate of $\mathrm{C} 97\left(10^{6} M_{\odot}\right)$. This mass obviously depends on the IMF used in the model, in this case a Salpeter IMF with masses between 1 and $100 M_{\odot}$, and would increase to $2.1 \times 10^{6} M_{\odot}$ if the mass distribution extends down to $0.1 M_{\odot}$. However, assuming a single slope value over the whole $0.1-$ $100 M_{\odot}$ range is probably not justified (see for instance the detailed study of Kroupa et al. 1993) and usually overestimates the stellar mass. Using a slope 1.25 between 0.1 and $1 M_{\odot}$ (Scalo 1986), the mass of the cluster becomes $1.2 \times 10^{6} M_{\odot}$. This mass is well in the range observed for SSCs, although compared to SBS 0335-052 or the Antennae, it appears on the low side (see Plante \& Sauvage 2002; Gilbert et al. 2000).

Concerning the geometry of the cocoon around the cluster, we find that it extends out to a radius of $\sim 140 \mathrm{pc}$, or approximately $9^{\prime \prime}$. This value strongly depends on $T_{\text {in }}$ and it is smaller for larger values of the inner temperature. We point out that this is the predicted size of the dust cloud inside which the cluster should reside, but that depending on the observing wavelength, the angular size of the source will vary, since the dust temperature strongly decreases with radius. Even though, this is rather large compared to the size of the central star formation region of the galaxy (see Fig. 1). This can be resolved in a number of ways. (1) Cluster 5 is only located in projection in the central region, but it is in fact behind, or less likely in front of the star forming region, or (2) cluster 5 is indeed part of the star forming region, and the dust cloud we model here encompasses it all. In that second case, which is more likely, all the central clusters should suffer from some extinction, which is the case, and all these provide some heating to the dust, so that our SED for cluster 5 is overestimated. Correcting this is impossible, but would lead to a lower luminosity for the central cluster and a smaller stellar mass. We note though, that the high resolution observations in the $10-20 \mu \mathrm{m}$ region show that only cluster 5 contributes infrared emission. This means that the heating provided by the other clusters is likely small, hence the correction on the model result should be small as well. It is interesting to note that the predicted size should be close to the observed size in the submillimeter range, since in that range we are most sensitive to cold dust. Indeed the SCUBA map of James et al. (2002) shows a source with a compatible angular size (25-30" diameter at the $6 \sigma$ level).

The most striking properties of the dust cocoon, as found from the best-fitting model, are directly connected to the dust grains. We find that the exponent of the grain size distribution is 2.5 , shallower than in the MRN distribution, i.e. it biases the distribution toward larger grains compared to the standard interstellar medium, as was found for SBS 0335-052. As noted in Plante \& Sauvage (2002) this appears to be common in high density regions exposed to strong radiation fields (see for instance the work of Maiolino et al. 2001). With sizes between $0.005 \mu \mathrm{m}$ and $0.5 \mu \mathrm{m}$, we do not find in NGC 5253 the lack of small grains that was observed in SBS 0335-052 where sizes ranged between 0.022 and $1 \mu \mathrm{m}$. Finally the chemical composition of the dust is very different from that found in the general ISM of our galaxy: we find that for the best-fit model, the dust composition requires no silicate component, being made of $84 \%$ graphite and $16 \%$ amorphous carbon. This is an extreme composition, even more severe than in the SBS 0335-052 cases where the best fit model required 23\% silicates (Plante \& Sauvage 2002). It first finds some observational support in the SWS spectrum of Crowther et al. (1999): even though independent measurements show significant extinction toward cluster 5, the spectrum does not show any absorption at the location of the strongest silicate band, $9.7 \mu \mathrm{m}$. We have then explored the deterioration of the fit if we intentionnaly add silicates to the dust mixture, while keeping the rest of the model constant. We observe that the figure of merit of the fit degrades gradually, being twice as large as the best fit value for a $25 \%$ abundance of silicates. However the worst problem is that since our model requires hot grains toward the center of the structure to fit the NIR-MIR emission, as soon as the fraction of silicates reaches $10 \%$, we start to observe emission features in the model, which are absent from the SWS spectrum for instance. We therefore feel that indeed the dust in the cocoon presents a severe lack of silicates.

We find that the density profile along the shell radius is shallower than in SBS 0335-052. Here the shell has a flat profile over the first $2-5 \%$ of its radius and then falls with an exponent of $1 / 2$. This profile, combined with the dust composition and size distribution, leads to a total dust mass in the envelope of $1.5 \times 10^{5} M_{\odot}$. The dust mass is directly proportional to the optical depth. It obviously also depends on the chemical composition, dust size distribution and density profile, all three parameters also influencing the optical depth. We find that the acceptable range of values can lead to a variation of the dust mass by a factor of $\sim 2$. Meier et al. (2002) estimate that $\sim 10^{7} M_{\odot}$ of $\mathrm{H}_{2}$ is present in the central region of the galaxy. Not all this gas is associated to the star formation episode: if we follow the estimation of the same authors, only $5 \times 10^{5} M_{\odot}$ of $\mathrm{H}_{2}$ are causally connected to the current starburst, which obviously includes cluster 5 . This would imply a molecular gas to dust mass ratio of $\sim 3-4$. This is rather high, even considering the lack of good statistics on this particular ratio. It is however not possible to elaborate on this particular issue, given the facts that (1) the molecular mass comes from an interferometric measurement, (2) NGC 5253 is a low-metallicity star-forming galaxy where the $\mathrm{CO}-$ to- $\mathrm{H}_{2}$ conversion ratio can be quite different from the standard value and (3) the determination of which fraction of the $\mathrm{H}_{2}$ gas mass is actually linked with the current burst is a difficult matter. We also note here that the mass of dust would be decreased if DUSTY allowed for temperature fluctuations in the dust (see Plante \& Sauvage 2002). If we consider that the dust mass in the cocoon is a good measure of the total dust mass in the galaxy (the cocoon provides almost all the IR-Submm luminosity), then the molecular gas to dust mass ratio in the central region of the galaxy becomes $\sim 70$, i.e. a more reasonable value.

Finally, we note that in a recent investigation of the ionized nebula associated with cluster 5, Turner et al. (2003) consider that none of the optical clusters, those detected in the HST data, coincide with the near-infrared source. This rests on a displacement between cluster 5 in the HST data and the NIR main source of $0 ! 3 \pm 0$ ' 1 , which we also confirm with our data. Does this mean that indeed the two objects, cluster 5 , and the infrared 
source are different? We think this is too small an offset to guarantee that indeed we have two independent sources here. The visual location of the source could for instance be affected by non-uniform extinction on the face of the cluster. To be complete however we investigated the consequences of Turner et al. (2003) statement, which basically results in the HST data dropping out of the fit. The main consequence is that the optical depth has to increase to values in the range 15-20, which in turn increases the dust mass required in the envelope. We therefore conclude that the model we have presented here represents at least a lower limit (in terms of the mass involved) to the actual situation in NGC 5253. To be able to state whether we are dealing with a single source from the visible to the NIR or not would require high-resolution images covering the $V$ to $K$ band, in order to "follow" the source's morphology and position with wavelength.

\section{Conclusions}

We can summarize the conclusions of our work as follows:

- We have obtained very high quality NIR data of NGC 5253 in the $K \mathrm{~s}$ and $L^{\prime}$ bands. The optically bright clusters detected all show a red excess that can be explained with the combined effect of extinction and dust emission. This however must be considered cautiously for the clusters in the red supergiant phase due to the uncertainty introduced on the models by the metallicity.

- One single cluster dominates the galaxy output beyond $3 \mu \mathrm{m}$. We have detected NGC 5253 at $1.2 \mathrm{~mm}$, we attribute the flux observed at this wavelength to the IR bright cluster known as cluster 5 .

- Using our observations, archive data and data available in the literature we have built a complete spectral energy distribution from the optical to the millimeter, doing this we find further evidence that one single cluster is responsible for most of the IR flux which is comparable to the optical flux of the whole galaxy.

We have then used the model DUSTY to fit the SED and derive the parameters of the IR cluster. We have found that:

- The IR cluster has a stellar mass of about $0.82-2.1 \times 10^{6} M_{\odot}$ depending on the IMF used, and a luminosity equivalent to $4700 \mathrm{O} 7 \mathrm{~V}$ stars. This cluster is obscured by 7 mag of optical extinction.

- The dust in which the cluster is embedded shows the now familiar bias toward shallower size distribution than the MRN one, and strikingly lacks silicates, a composition that can explain the absence of a significant silicate absorption band. Adding silicates to the best fit model, to obtain a dust composition that resembles the classical one, lead to emission features in the model spectrum as the NIR part of the SED requires relatively hot grains. None of these features are observed.

- The total dust mass necessary to reproduce the optical to submm SED of cluster 5 is $1.5 \times 10^{5} M_{\odot}$. This is a relatively small amount intrinsically, although when compared to the molecular mass observed to be involved in the current episode of star formation, it becomes a substantial fraction of the ISM.

Finally we wish to stress again that NGC 5253 provides a striking example of an object where one part of the spectral energy distribution, i.e. the IR-submm one, is completely decoupled from the other, the UV-optical one. The existence of such objects, if not a space oddity, would cast some doubt on our ability to predict the global spectral energy distributions of galaxies as they evolve in time.

Acknowledgements. We are grateful to Daniela Calzetti, Claus Leitherer and Stéphanie Plante for providing us useful information during our work. We thank Daniel Schaerer and Leticia MartinHernández for letting us quote their new NGC 5253's Q1-band flux. We also thank the ESO staff that carried out the observations at the VLT in Service Mode providing us data of outstanding quality. MS acknowledges the support of ESO under the Visiting Scientist program. This research has made use of the NASA/IPAC Extragalactic Database (NED) which is operated by the Jet Propulsion Laboratory, California Institute of Technology, under contract with the National Aeronautics and Space Administration. The ISO data used in this paper was processed with CIA, a joint development by the ESA Astrophysics Division and the ISOCAM consortium led by the ISOCAM P.I. C. Cesarsky, Direction des Sciences de la Matière, CEA Saclay, France. Finally we thank the referee, Richard de Grijs, for his comments which contributed to improve this paper and for his very positive attitude and availaibility to discussion.

\section{References}

Beck, S. C., Turner, J. L., Ho, P. T. P., Lacy, J. H., \& Kelly, D. 1996, ApJ, 457, 610

Caldwell, N., \& Phillips, M. M. 1989, ApJ, 338, 789

Calzetti, D., Meurer, G. R., Bohlin, R. C., et al. 1997, AJ, 114, 1834 (C97)

Calzetti, D., Conselice, C. J., Gallagher, J. S., \& Kinney, A. L. 1999, AJ, 118, 797

Chapman, S. C., Helou, G., Lewis, G. F., \& Dale, D. A. 2003, ApJ, 588, 186

Chary, R. R., \& Elbaz, D. 2001, ApJ, 556, 562

Conti, P. S., \& Vacca, W. D. 1994, ApJ, 423, L97

Contursi, A., Lequeux, J., Cesarsky, D., et al. 2000, A\&A, 362, 310

Coulais, A., \& Abergel, A. 2000, A\&AS, 141, 533

Crowther, P. A., Beck, S. C., Willis, et al. 1999, MNRAS, 304, 654

Dale, D. A., \& Helou, G. 2002, ApJ, 576, 159

Draine, B. T., \& Lee, H. M. 1984, ApJ, 285, 89

Forbes, D. A., Ward, M. J., Depoy, D. L., Boisson, C., \& Smith, M. S. 1992, MNRAS, 254, 509

Frogel, J. F., Elias, J. H., \& Phillips, M. M. 1982, ApJ, 260, 70

Gibson, B. K., Stetson, P. B., Freedman, W. L., et al. 2000, ApJ, 529, 723

Gilbert, A. M., Graham, J. R., McLean, I. S., et al. 2000, ApJ, 533, L57

Glass, I. S. 1973, MNRAS, 164, 155

Gorjian, V. 1996, AJ, 112, 1886

Gorjian, V., Turner, J. L., \& Beck, S. C. 2001, ApJ, 554, L29

Hunt, L. K., Vanzi, L., \& Thuan, T. X. 2001, A\&A, 377, 66 
Ivezić, Ž., \& Elitzur, M. 1997, MNRAS, 287, 799

James, A., Dunne, L., Eales, S., \& Edmunds, M. G. 2002, MNRAS, 335,753

Kobulnicky, H. A., \& Johnson, K. E. 1999, ApJ, 527, 154

Kobulnicky, H. A., Kennicutt, R. C., \& Pizagno, J. L. 1999, ApJ, 514, 544

Kroupa, P., Tout, C. A., \& Gilmore, G. 1993, MNRAS, 262, 545

Langer, N., \& Maeder, A. 1995, A\&A, 297, 483

Lebofsky, M. J., \& Rieke, G. H. 1979, ApJ, 229, 111

Leitherer, C., Schaerer, D., Goldader, J. D., et al. 1999, ApJS, 123, 3

Maiolino, R., Marconi, A., \& Oliva, E. 2001, A\&A, 365, 37

Martin, C. L., \& Kennicutt, R. C. 1995, ApJ, 447, 171

Martin-Hernandez, N. L., et al. 2004, in preparation

Mathis, J. S., Rumpl, W., \& Nordsieck, K. H. 1977, ApJ, 217, 425

Meier, D. S., Turner, J. L., \& Beck, S. C. 2002, AJ, 124, 877

Moorwood, A. F. M., \& Glass, I. S. 1982, A\&A, 115, 84

Moshir, M., Kopan, G., Conrow, T., et al. 1990, Infrared Astronomical Satellite Catalogs, The Faint Source Catalog, version 2.0, http://irsa.ipac.caltech.edu/IRASdocs/surveys/ fsc.html
Origlia, L., Goldader, J. D., Leitherer, C., Schaerer, D., \& Oliva, E. 1999, ApJ, 514, 96

Plante, S., \& Sauvage, M. 2002, AJ, 124, 1995

Rieke, G. H., \& Low, F. J. 1972, ApJ, 176, L95

Roche, P. F., Aitken, D. K., Smith, C. H., \& Ward, M. J. 1991, MNRAS, 248, 606

Scalo, J. M. 1986, Fund. Cosm. Ph., 11, 1

Thim, F., Tamman, G. A., Saha, A., et al. 2003, ApJ, 590, 256

Thuan, T. X., Izotov, I. Y., \& Lipovetsky, V. A. 1997, ApJ, 477, 661

Thuan, T. X., Sauvage, M., \& Madden, S. C. 1999, ApJ, 516, 783

Turner, J. L., Beck, S. C., \& Hurt, R. L. 1997, ApJ, 474, L11

Turner, J. L., Ho, P. T. P., \& Beck, S. 1998, AJ, 116, 1212

Turner, J. L., Beck, S. C., \& Ho, P. T. P. 2000, ApJ, 532, L109

Turner, J. L., Beck, S. C., Crosthwhaite, L. P., et al. 2003, Nature, 423, 621

Vacca, W. D., Garmany, C. D., \& Shull, J. M. 1996, ApJ, 460, 914

Vacca, W. D., Johnson, K. E., \& Conti, P. S. 2002, AJ, 123, 772

Vanzi, L. 2003, A\&A, 408, 523

Van den Bergh, S. 1980, PASP, 92, 122

Welch, G. A. 1970, ApJ, 161, 821

Witt, A. N., Thronson, H. A., \& Capuano, J. M. 1992, ApJ, 393, 611 